\title{
COMPRESSIVE SAMPLING WITH UNKNOWN BLURRING FUNCTION: APPLICATION TO PASSIVE MILLIMETER-WAVE IMAGING
}

\author{
Bruno Amizic $^{1}$, Leonidas Spinoulas ${ }^{1}$, Rafael Molina $^{2}{ }^{*}$, Aggelos K. Katsaggelos ${ }^{1 \dagger}$ \\ ${ }^{1}$ Dept. of Electrical Engineering and Comp. Sc., Northwestern University, Evanston, IL 60208, USA \\ 2 Dept. de Ciencias de la Computación e I.A., Universidad de Granada, 18071 Granada, Spain
}

\begin{abstract}
We propose a novel blind image deconvolution (BID) regularization framework for compressive passive millimeter-wave (PMMW) imaging systems. The proposed framework is based on the variablesplitting optimization technique, which allows us to utilize existing compressive sensing reconstruction algorithms in compressive BID problems. In addition, a non-convex $l_{p}$ quasi-norm with $0<p<1$ is employed as a regularization term for the image, while a simultaneous auto-regressive (SAR) regularization term is utilized for the blur. Furthermore, the proposed framework is very general and it can be easily adapted to other state-of-the-art BID approaches that utilize different image/blur regularization terms. Experimental results, obtained with simulations using a synthetic image and real PMMW images, show the advantage of the proposed approach compared to existing ones.
\end{abstract}

Index Terms - Variable-splitting, inverse methods, compressive sensing, blind image deconvolution.

\section{INTRODUCTION}

Passive millimeter-wave imagers (PMMWI) are imaging devices that collect natural millimeter-wave radiation from the scene. The literature on passive millimeter-wave imaging technology is rich (see [1] for a review). The millimeter-wave regime is part of the electromagnetic spectrum in the frequency band between 30 and $300 \mathrm{GHz}$, and has significant advantages over the conventional (e.g., visible light and infrared) imaging devices in low-visibility conditions during day or night. In environments dominated by clouds, fog, smoke, rain, snow, and dust-storms, the millimeter-wave radiation is attenuated a few orders of magnitude less than the visual or infrared radiation. The ability to capture the scene in low-visibility conditions has led to numerous applications of passive millimeter-wave technology over the course of years [1]. Furthermore, advances in millimeter-wave radiometry (e.g., [2]) and integrated-circuit technologies (e.g., [3]) extended their use while technological breakthroughs led to the development of passive-millimeter wave video devices (e.g., [4]). More recently, active and passive millimeterwave scanners have been successfully used in airports to detect a broad range of concealed threats (e.g., [5]).

This paper is organized as follows. We introduce the modeling of compressive PMMW imaging in Section 2. In Section 3, we provide an overview of the existing compressive PMMW reconstruction algorithms, and summarize the concepts of variable-splitting optimization techniques. The proposed compressive BID algorithm is

\footnotetext{
* This work was supported in part by the "Ministerio de Ciencia e Innovación" under contract TIN2010-15137.

$\dagger$ This work was supported in part by the Department of Energy.
}

analyzed in Section 4. Finally, supporting experimental results are provided in Section 5, and conclusions in Section 6.

\section{COMPRESSIVE PASSIVE MILLIMETER-WAVE IMAGING}

Conventional PMMW imaging systems consist of two main components [6]. The first is responsible for measuring the incoming radiation while the second is responsible for directing the measurements and forming the image. The main disadvantage of these early devices is their complexity and their long acquisition time. Recently, several imaging systems have been proposed $[6,7]$ to overcome these problems. They utilize the concepts of Compressive Sensing (CS) theory. In a CS setting it is shown that far fewer measurements, than the desired resolution, are needed to accurately reconstruct signals that are sparse in a transformed domain [8]. The standard formulation of the compressive passive millimeter-wave imaging model is given in matrix-vector form by

$$
\mathbf{y}=\mathbf{\Phi} \mathbf{x}+\mathbf{n}
$$

where the $N \times 1$ vector $\mathbf{x}$ and the vector $\mathbf{n}$ of size $M \times 1$ represent the lexicographically ordered unknown image and the observation noise respectively. The $M \times N$ matrix $\boldsymbol{\Phi}$ represents the measurement matrix, and the $M \times 1$ vector $\mathbf{y}$ represents the observation vector. The unknown image, $\mathbf{x}$, is assumed to be of size $m \times n=N$. Note that $M<<N$.

In this paper we modify the acquisition model in (1) by including the blurring function, which is a more realistic modeling assumption due to the blurring introduced by the imaging system lens (defocusing, lens imperfections). Therefore, in matrix-vector form, the formulation of the compressive PMMW imaging model with unknown blurring function is modeled as

$$
\mathbf{y}=\boldsymbol{\Phi} \mathbf{H x}+\mathbf{n},
$$

where the $N \times N$ matrix $\mathbf{H}$ represents the blurring matrix created from the blurring point spread function (denoted by $\mathbf{h}$ from now on).

In order to utilize state-of-the-art compressive sensing algorithms, in their original forms, it is advantageous to rewrite (2) as

$$
\mathbf{y}=\boldsymbol{\Phi} \mathbf{W a}+\mathbf{n},
$$

where the $N \times 1$ vector a represents the transformed coefficients corresponding to $N$ basis vectors that span the column space of the $N \times N$ matrix $\mathbf{W}$, such that $\mathbf{W a}=\mathbf{H x}$. 


\section{EXISTING COMPRESSIVE SENSING RECONSTRUCTION ALGORITHMS}

Compressive sensing image recovery calls for calculating the sparse vector a from the noisy observation $\mathbf{y}$ in (3). It is widely known that this is an ill-conditioned problem, and that simple recovery algorithms based on least-squares techniques are unable to accurately recover the unknown sparse vector a. Instead, the most commonly used approach is to solve the following optimization problem,

$$
\min _{\mathbf{a}}\|\mathbf{y}-\mathbf{\Phi} \mathbf{W a}\|^{2}+\tau\|\mathbf{a}\|_{1}
$$

where $\|\cdot\|$ denotes the Euclidean norm, $\|\cdot\|_{1}$ denotes the $l_{1}$-norm, and $\tau$ is non-negative regularization parameter. In the last few years numerous approaches have been proposed to solve this optimization scheme; for example, linear programing methods (e.g., [9]), statistical methods (e.g., [10]), interior-point methods (e.g., [11]), and Bayesian methods (e.g., [7]). Finding efficient and accurate solutions for the sparse vector, $\mathbf{a}$, is still a very active research problem. In this paper our focus is to establish a robust framework in which the existing state-of-the-art CS algorithms can be efficiently utilized to solve the compressed blind image deconvolution problem as defined in (2), where both image (x) and blur (h) are unknown. This goal is accomplished by employing variable-splitting concepts from optimization theory [12]. The developed model will be applied to PMMW images.

\subsection{Overview of Variable-Splitting Techniques}

Recently, variable-splitting techniques have been used in numerous image processing applications (e.g., $[13,14])$. The objective of variable-splitting approaches is to introduce hidden variables to the original optimization problem, in order to simplify optimization of the functionals (e.g., [13]), or utilize some existing state-of-the-art algorithms (e.g., [15]). In general, as shown in [12,13], optimization problems of the form

$$
\min _{\mathbf{x}} R_{1}(\mathbf{x})+R_{2}(f(\mathbf{x}))
$$

where $R_{1}(\cdot)$ and $R_{2}(\cdot)$ are some regularization functionals and $f(\cdot)$ denotes a vector function, can be solved by finding the solution to the following optimization problem

$$
\min _{\mathbf{x}, \mathbf{u}} R_{1}(\mathbf{x})+\frac{\eta}{2}\|\mathbf{u}-f(\mathbf{x})\|^{2}+R_{2}(\mathbf{u})
$$

and by letting $\eta$ approach $\infty$.

\section{PROPOSED APPROACH}

Many existing BID approaches are formulated as optimization problems (see [16] for the most recent literature review), where a cost function, with a number of regularization constraint terms, is minimized. The cost function is chosen as the error function $\|\mathbf{y}-\mathbf{H x}\|^{2}$ which ensures fidelity to the data. The regularization terms are used to impose additional constraints on the optimization problem. Generally, these constraints ensure image and blur smoothness, that is, their high frequency energies are minimized. The effect of the regularization terms is controlled by regularization parameters, which basically represent the trade-off between fidelity to the data and the desirable properties (smoothness) of the solution. A general form of the functional to be minimized with such approaches, adapted to our imaging model in (2), is given by,

$$
\min _{\mathbf{x}, \mathbf{h}} \frac{\beta}{2}\|\mathbf{y}-\mathbf{\Phi H \mathbf { x }}\|^{2}+\alpha R_{1}(\mathbf{x})+\gamma R_{2}(\mathbf{h}),
$$

where $\beta, \alpha$, and $\gamma$ are non-negative regularization parameters. Let us now propose a novel unconstrained optimization problem that exploits concepts from both variable-splitting and compressive sensing approaches. From (6), with the assumption that the hidden vector $\mathbf{u}$ is compressible in a transformed domain (i.e., $\mathbf{u}=\mathbf{W a}$ ), it follows that the optimization problem in (7) can be reformulated as

$$
\min _{\mathbf{x}, \mathbf{h}, \mathbf{a}} \frac{\beta}{2}\|\mathbf{y}-\mathbf{\Phi} \mathbf{W} \mathbf{a}\|^{2}+\frac{\eta}{2}\|\mathbf{W a}-\mathbf{H} \mathbf{x}\|^{2}+\alpha R_{1}(\mathbf{x})+\gamma R_{2}(\mathbf{h}) .
$$

Finally, in order to increase the modeling robustness, and assuming that the hidden image $\mathbf{u}$ is sparse in a transformed domain, we impose a sparse constraint on the transformed coefficients and propose the following unconstrained blind image deconvolution algorithm for compressive sensing

$$
\begin{aligned}
\hat{\mathbf{x}}, \hat{\mathbf{h}}, \hat{\mathbf{a}}=\underset{\mathbf{x}, \mathbf{h}, \mathbf{a}}{\operatorname{argmin}} & \left\{\frac{\beta}{2}\|\mathbf{y}-\mathbf{\Phi} \mathbf{W} \mathbf{a}\|^{2}+\frac{\eta}{2}\|\mathbf{W a}-\mathbf{H} \mathbf{x}\|^{2}+\right. \\
& \left.+\alpha R_{1}(\mathbf{x})+\gamma R_{2}(\mathbf{h})+\tau\|\mathbf{a}\|_{1}\right\} .
\end{aligned}
$$

A widely used approach to solve the above problem is alternating minimization (AM) which follows the steepest descent with respect to one unknown while fixing the other unknowns. Clearly, AM approach sets an optimization framework in which we can utilize existing state-of-the-art compressive sensing methods when solving for the sparse vector $\mathbf{a}$.

Let us now specify the regularization terms utilized for the image and blur. We employ a variant of $l_{p}$ quasi-norm as a regularization term for the image,

$$
R_{1}(\mathbf{x})=\left[\sum_{d \in \mathrm{D}} 2^{1-o(d)} \sum_{i}\left|\Delta_{i}^{d}(\mathbf{x})\right|^{p}\right],
$$

where $o(d) \in\{1,2\}$ denotes the order of the difference operator $\Delta_{i}^{d}(\mathbf{x}), 0<p<1$, and $d \in \mathrm{D}=\{h, v, h h, v v, h v\} . \Delta_{i}^{h}(\mathbf{x})$ and $\Delta_{i}^{v}(\mathbf{x})$ correspond, respectively, to the horizontal and vertical first order differences, at pixel $i$, that is, $\Delta_{i}^{h}(\mathbf{x})=u_{i}-u_{l(i)}$ and $\Delta_{i}^{v}(\mathbf{x})=u_{i}-u_{a(i)}$, where $l(i)$ and $a(i)$ denote the nearest neighbors of $i$, to the left and above, respectively. The operators $\Delta_{i}^{h h}(\mathbf{x})$, $\Delta_{i}^{v v}(\mathbf{x}), \Delta_{i}^{h v}(\mathbf{x})$ correspond, respectively, to horizontal, vertical and horizontal-vertical second order differences, at pixel $i$. This type of regularizer for the image has also been used in [17].

In addition, we utilize a simultaneous auto-regressive (SAR) regularization for the blur,

$$
R_{2}(\mathbf{h})=\|\mathbf{C h}\|^{2},
$$

where $\mathbf{C}$ is the Laplacian operator. The SAR regularization (see [18] for a Bayesian interpretation) imposes smoothness on the blur estimates, and it is typically applied as a model for blurs that are expected to be smooth, as is the case of blurring functions for compressive PMMWI.

Furthermore, as the minimization of the non-convex functional in (9) is not straightforward, we employ the majorizationminimization approach [19] to bound the non-convex image regularizer $R_{1}(\mathbf{x})$ by the functional $\mathrm{M}_{1}(\mathbf{x}, \mathbf{V})$, that is,

$$
R_{1}(\mathbf{x}) \leq \mathrm{M}_{1}(\mathbf{x}, \mathbf{V})
$$


where $M_{1}(\mathbf{x}, \mathbf{V})$ is defined as

$$
\mathrm{M}_{1}(\mathbf{x}, \mathbf{V})=\frac{p}{2} \sum_{d \in \mathrm{D}} 2^{1-o(d)} \sum_{i} \frac{\left[\Delta_{i}^{d}(\mathbf{x})\right]^{2}+\frac{2-p}{p} v_{d, i}}{v_{d, i}^{1-p / 2}}
$$

and $\mathbf{V}$ is a matrix with elements $v_{d, i}>0$. Detailed derivation of this bound is presented in [20].

Finally, we present our blind image deconvolution algorithm for compressive sampling, which will be later applied to PMMW images. In this algorithm we follow a general framework for solving variable-splitting based optimization problems (see Section 17.2 in [12] for more details).

Algorithm. Given $\alpha, \beta, \gamma, \tau, \eta^{1}, \mathbf{a}^{1}$, and $\mathbf{V}^{1}$, where the rows of $\mathbf{V}^{k}$ are denoted by $\mathbf{v}_{d}^{k} \in\left(R^{+}\right)^{N}$, with $d \in\{h, v, h h, v v, h v\}$, and an initial estimate of the blur $\mathbf{h}^{1}$.

For $k=1,2, \ldots$ until a stopping criterion is met:

\section{Calculate}

$$
\begin{aligned}
\mathbf{x}^{k} & =\left[\eta^{k}\left(\mathbf{H}^{k}\right)^{t}\left(\mathbf{H}^{k}\right)+\alpha p \sum_{d} 2^{1-o(d)}\left(\boldsymbol{\Delta}^{d}\right)^{t} \mathrm{~B}_{d}^{k}\left(\boldsymbol{\Delta}^{d}\right)\right]^{-1} \\
& \times \eta^{k}\left(\mathbf{H}^{k}\right)^{t} \mathbf{W} \mathbf{a}^{k}
\end{aligned}
$$

where $\mathrm{B}_{d}^{k}$ is a diagonal matrix with entries $\mathrm{B}_{d}^{k}(i, i)=$ $\left(v_{d, i}^{k}\right)^{p / 2-1}$ and $\boldsymbol{\Delta}^{d}$ is the convolution matrix of the difference operator $\Delta_{i}^{d}(\cdot)$.

\section{Calculate}

$$
\mathbf{h}^{k+1}=\left[\eta^{k}\left(\mathbf{X}^{k}\right)^{t}\left(\mathbf{X}^{k}\right)+\gamma \mathbf{C}^{t} \mathbf{C}\right]^{-1} \times \eta^{k}\left(\mathbf{X}^{k}\right)^{t} \mathbf{W} \mathbf{a}^{k},
$$

where $\mathbf{X}^{k}$ is the convolution matrix of the image $\mathbf{x}^{k}$.

3. Find

$$
\begin{aligned}
\mathbf{a}^{k+1} & =\underset{\mathbf{a}}{\operatorname{argmin}} \frac{\beta}{2}\|\mathbf{y}-\mathbf{\Phi} \mathbf{W a}\|^{2} \\
& +\frac{\eta^{k}}{2}\left\|\mathbf{W a}-\mathbf{H}^{k+1} \mathbf{x}^{k}\right\|^{2}+\tau\|\mathbf{a}\|_{1},
\end{aligned}
$$

4. For each $d \in\{h, v, h h, v v, h v\}$ calculate

$$
v_{d, i}^{k+1}=\left[\Delta_{i}^{d}\left(\mathbf{x}^{k}\right)\right]^{2},
$$

5. Set

$$
\eta^{k+1}=\theta \eta^{k}
$$

where $\theta>1$.

Set

$$
\hat{\mathbf{a}}=\lim _{k \rightarrow \infty} \mathbf{a}^{k}, \hat{\mathbf{h}}=\lim _{k \rightarrow \infty} \mathbf{h}^{k}, \hat{\mathbf{x}}=\lim _{k \rightarrow \infty} \mathbf{x}^{k},
$$

Note that (16) can be re-written as

$$
\mathbf{a}^{k+1}=\underset{\mathbf{a}}{\operatorname{argmin}}\left\|\mathbf{y}^{\prime}-\mathbf{\Phi}^{\prime} \mathbf{W a}\right\|^{2}+\tau\|\mathbf{a}\|_{1},
$$

where $\mathbf{y}^{\prime}=\left[\begin{array}{c}\sqrt{\frac{\beta}{2}} \mathbf{y} \\ \sqrt{\frac{\eta^{k}}{2}} \mathbf{H}^{k+1} \mathbf{x}^{k}\end{array}\right]$ and $\boldsymbol{\Phi}^{\prime}=\left[\begin{array}{c}\sqrt{\frac{\beta}{2}} \boldsymbol{\Phi} \\ \sqrt{\frac{\eta^{k}}{2}} \mathbf{I}\end{array}\right]$. Therefore, when solving for the sparse vector a, we can utilize existing compressive sensing reconstruction algorithms (e.g., [11]).

\section{EXPERIMENTAL RESULTS}

We evaluate the performance of the proposed algorithm on both a synthetic image (which is similar in structure to real PMMW images) and real PMMW images. The size of test images is $48 \times 48$, as this is a typical block size for the PMMW images [6]. In this paper, we adopted the peak signal to noise ratio (PSNR) as a comparison metric, which is defined as

$$
\mathrm{PSNR}=10 \log _{10} \frac{N L^{2}}{\|\mathbf{x}-\hat{\mathbf{x}}\|^{2}},
$$

where $\mathbf{x}$ and $\hat{\mathbf{x}}$ are the original and estimated images, respectively, and the constant $L$ represents the maximum possible intensity value in image $\mathbf{x}$. The proposed algorithm is terminated when the convergence criterion $\left\|\mathbf{x}^{k}-\mathbf{x}^{k-1}\right\| /\left\|\mathbf{x}^{k-1}\right\|<10^{-4}$ is satisfied or if the number of iterations reaches 20 . The rest of the algorithmic parameters, $p$ and $\theta$, are set to 0.8 and 2.0, respectively. Furthermore, we initialize the proposed algorithm with a Gaussian blur of variance 5 for all test cases that follow.

In the first experiment, we blur a synthetic image (i.e., SheppLogan phantom) with a Gaussian blur of variance 3, and perform CS measurements using both Gaussian and Binary measurement matrices at the compressive ratios of $0.2,0.4,0.6,0.8$, and 1.0. In addition, the parameters $\alpha, \beta, \gamma$, and $\tau$ are found by exhaustive search through the unknown parameter space for each compressive ratio (i.e., $M / N$ ). Finally, we add observation noise so that the signalto-noise ratio (SNR) equals $40 \mathrm{~dB}$. From Table 1, it is clear that the proposed algorithm is very robust as we can restore the image at various compressive ratios. In addition, by constraining the blur support after each iteration, we can further improve the restoration quality. Example restorations from Table 1 are shown in Figure 1. Also, in Figure 1 we show the comparison with the method proposed in [20] $(\mathrm{PSNR}=17.16 \mathrm{~dB})$, where we assume that $\boldsymbol{\Phi}$ is the identity matrix. In [20], the authors compared different non-blind and blind image restoration methods and show that their method is competitive to state-of-the-art (see [20] for more details). The performance of the proposed method, at different compressive ratios, competes with the method in [20]. The advantage of the proposed approach is evident as we can achieve similar restoration quality with far less measurements than the number of pixels in the original image $\mathbf{x}$.

Table 1: PSNR values for the Shepp-Logan image degraded by a Gaussian blur of variance 3 at $S N R=40 \mathrm{~dB}$.

\begin{tabular}{|c|c|c|c|}
\hline \multirow{2}{*}{ Measurement Matrix } & \multirow{2}{*}{ Compressive Ratio } & \multicolumn{2}{|c|}{ Blur Support } \\
\cline { 3 - 4 } & & $11 \times 11$ & $48 \times 48$ \\
\hline \hline \multirow{3}{*}{ Gaussian } & 1.0 & 17.62 & 17.14 \\
& 0.8 & 17.54 & 17.12 \\
& 0.6 & 17.12 & 16.88 \\
& 0.4 & 16.62 & 16.19 \\
& 0.2 & 15.74 & 15.02 \\
\hline \multirow{3}{*}{ Binary } & 1.0 & 17.32 & 17.00 \\
& 0.8 & 17.22 & 16.91 \\
& 0.6 & 17.14 & 16.90 \\
& 0.4 & 16.62 & 16.11 \\
& 0.2 & 16.07 & 15.50 \\
\hline
\end{tabular}

In the second experiment we evaluate the performance of the proposed algorithm using real PMMW images (from [6]), and show the restoration results using random Gaussian measurement matrices in Figure 2. In this experiment the parameters $\alpha, \beta, \gamma$, and $\tau$ are set to $12.8,19 e 3,70 e 4$, and 100 , respectively. Also, we assume a priori that the size of the blur is $11 \times 11$, and re-enforce symmetry on the 

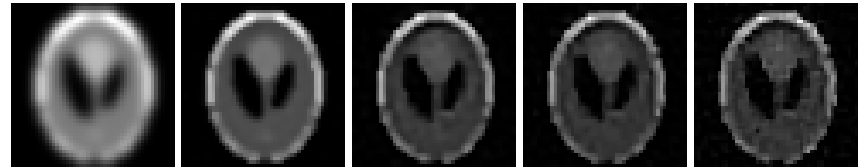

Fig. 1: Example restorations: $1^{\text {st }}$ column represents uncompressed blurred Shepp-Logan image; $2^{\text {nd }}$ column represents the restoration obtained by the method in [20] $; 3^{\text {rd }}, 4^{\text {th }}$, and $5^{\text {th }}$ columns represent, respectively, the restorations obtained by the proposed algorithm using $100 \%, 80 \%$, and $60 \%$ of the measurements, where $\boldsymbol{\Phi}$ is a Gaussian random matrix.

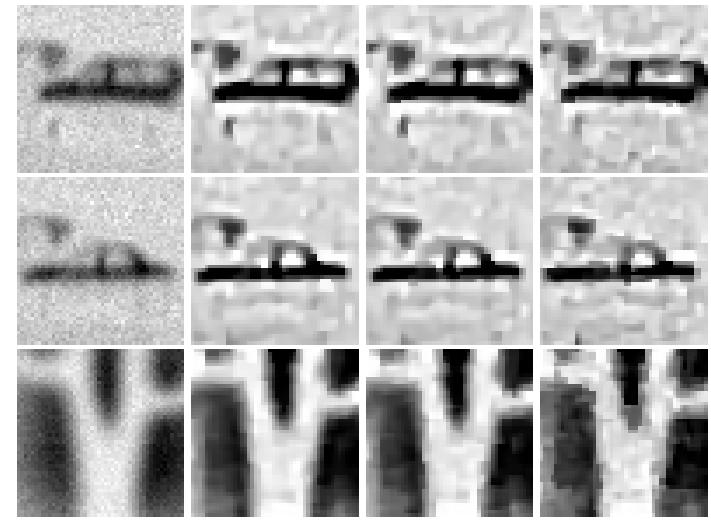

Fig. 2: Example restorations: $1^{\text {st }}$ column represents three different uncompressed blurred observations from [6]; $2^{\text {nd }}, 3^{\text {rd }}$, and $4^{\text {th }}$ columns represent, respectively, the restorations obtained by the proposed algorithm using $80 \%, 60 \%$, and $40 \%$ of the measurements, where $\boldsymbol{\Phi}$ is a Gaussian random matrix.

blur estimate after each iteration. It is evident that restorations of high visual quality can be obtained using the proposed method. In addition, the blurred-uncompressed image in Figure 2 corresponds to the best achievable restoration when the blurring function is not taken into consideration.

\section{CONCLUSIONS}

In this paper we presented a novel blind image deconvolution (BID) framework for imaging systems that are based on the principles of compressive sensing. The proposed algorithm employs variablesplitting optimization techniques, and existing compressive sensing state-of-the-art algorithms that cannot easily be extended to BID framework (i.e., when the blurring matrix is different from identity). In addition, the proposed framework is general, as can be seen in (9), and can easily be extended to other BID algorithms that utilize different regularization terms for the image and blur. Restorations of both a synthetic image and real PMMW images are presented to demonstrate the robustness of our approach. We clearly show that modeling of the unknown blur is desirable when restoring images obtained through lens-based CS imaging systems.

\section{REFERENCES}

[1] L. Yujiri, M. Shoucri, and P. Moffa, "Passive millimeter wave imaging," vol. 4, no. 3, pp. 39-50, 2003.
[2] R. H. Dicke, "The measurement of thermal radiation at microwave frequencies," Review of Scientific Instruments, vol. 17, no. 7, pp. 268-275, 1946.

[3] H. P. Moyer, J. J. Lynch, J. N. Schulman, R. L. Bowen, J. H. Schaffner, A. K. Kurdoghlian, and T. Y. Hsu, "A low noise chipset for passive millimeter wave imaging," in Proc. IEEE/MTT-S Int. Microwave Symp, 2007, pp. 1363-1366.

[4] J. A. Lovberg, C. Martin, and V. Kolinko, "Video-rate passive millimeter-wave imaging using phased arrays," in Proc. IEEE/MTT-S Int. Microwave Symp, 2007, pp. 1689-1692.

[5] O. Martinez, L. Ferraz, X. Binefa, I. Gomez, and C. Dorronsoro, "Concealed object detection and segmentation over millimetric waves images," in Proc. IEEE Computer Society Conf. Computer Vision and Pattern Recognition Workshops (CVPRW), 2010, pp. 31-37.

[6] N. Gopalsami, S. Liao, T. Elmer, A. Heifetz, and A. C. Raptis, "Compressive sampling in active and passive millimeter-wave imaging," in Proc. 36th Int Infrared, Millimeter and Terahertz Waves (IRMMW-THz) Conf, 2011, pp. 1-2.

[7] S. D. Babacan, M. Luessi, L. Spinoulas, A. K. Katsaggelos, N. Gopalsami, T. Elmer, R. Ahern, S. Liao, and A. Raptis, "Compressive passive millimeter-wave imaging," in Proc. 18th IEEE Int Image Processing (ICIP) Conf, 2011, pp. 2705-2708.

[8] E. J. Candes and M. B. Wakin, "An introduction to compressive sampling," vol. 25, no. 2, pp. 21-30, 2008.

[9] S. S. Chen, D. L. Donoho, and M. A. Saunders, "Atomic decomposition by basis pursuit," SIAM Journal on Scientific Computing, vol. 20, no. 1, pp. 33-61, 1998.

[10] R. Tibshirani, "Regression shrinkage and selection via the lasso," Journal of the Royal Statistical Society, Series B, vol. 58, pp. 267-288, 1994.

[11] S.-J. Kim, K. Koh, M. Lustig, S. Boyd, and D. Gorinevsky, "An interior-point method for large-scale 11-regularized least squares," vol. 1, no. 4, pp. 606-617, 2007.

[12] J. Nocedal and S. J. Wright, Numerical Optimization, Springer Series in Operations Research, 2006.

[13] J. M. Bioucas-Dias and M. A. T. Figueiredo, "Multiplicative noise removal using variable splitting and constrained optimization," vol. 19, no. 7, pp. 1720-1730, 2010.

[14] F. Sroubek, J. Kamenicky, and P. Milanfar, "Superfast superresolution," in Proc. 18th IEEE Int Image Processing (ICIP) Conf, 2011, pp. 1153-1156.

[15] H. Liao, F. Li, and M. K. Ng, "Selection of regularization parameter in total variation image restoration," J. Opt. Soc. Am. A, vol. 26, no. 11, pp. 2311-2320, 2009.

[16] T. E. Bishop, S. D. Babacan, B. Amizic, A. K. Katsaggelos, T. Chan, and R. Molina, Blind image deconvolution: problem formulation and existing approaches, CRC press, 2007.

[17] A. Levin, R. Fergus, F. Durand, and W. T. Freeman, "Image and depth from a conventional camera with a coded aperture," in SIGGRAPH '07: ACM SIGGRAPH 2007 papers, New York, NY, USA, 2007, p. 70, ACM.

[18] R. Molina, J. Mateos, and A. Katsaggelos, "Blind deconvolution using a variational approach to parameter, image, and blur estimation," vol. 15, no. 12, pp. 3715-3727, Dec. 2006.

[19] J. Bioucas-Dias, M. Figueiredo, and J. Oliveira, "Totalvariation image deconvolution: A majorization-minimization approach," in ICASSP'2006, 2006.

[20] B. Amizic, S. D. Babacan, R. Molina, and A. K. Katsaggelos, "Sparse bayesian blind image deconvolution with parameter estimation," in European Signal Processing Conference, Eusipco 2010. Aalborg, Denmark, August 2010, pp. 626-630. 